\title{
A psicoeducação no processo de trabalho do enfermeiro de saúde mental
}

\section{Psychoeducation in the work process of mental health nurses}

DOI: $10.46919 / \operatorname{archv3n3-001}$

Recebimento dos originais: 04/02/2022

Aceitação para publicação: 01/03/2022

\section{Mario Sergio Bruggmann}

Doutor em Enfermagem

Instituição: Universidade Federal de Santa Catarina Secretaria do Estado de Saúde do Estado de Santa Catarina

Endereço: Rua Esteves Júnior, 160, Centro, Florianópolis, Santa Catarina - Brasil

E-mail: mariobrugg@gmail.com

\section{Sandra Mara Corrêa}

Mestre em Enfermagem

Instituição: Universidade Federal de Santa Catarina Secretaria do Estado de Saúde do Estado de Santa Catarina

Endereço: Rua Esteves Júnior, 160, Centro, Florianópolis, Santa Catarina - Brasil

E-mail: sandramaraenf@yahoo.com.br

\section{Djéssica Korb}

Especialista em Enfermagem em Psiquiatria e Saúde Mental

Instituição: Secretaria do Estado de Saúde do Estado de Santa Catarina

Endereço: Rua Esteves Júnior, 160, Centro, Florianópolis, Santa Catarina - Brasil

E-mail: dje_korb@hotmail.com

\section{RESUMO}

Trata-se de uma revisão narrativa da literatura, cujo objetivo é descrever a psicoeducação enquanto técnica utilizada no processo de trabalho do enfermeiro de saúde mental. Foram realizadas buscas não sistemáticas de estudos, em abril e maio de 2020, sem recorte temporal, nas bases de dados LILACS, MEDLINE e SCOPUS. Foi evidenciado que a psicoeducação é uma técnica utilizada pelo enfermeiro de saúde mental em formato individual, através da consulta de enfermagem ou coletiva, por meio da formação de grupos. Nesta direção, constitui um fator cognitivo relevante para a modificação do pensamento e comportamento em usuários assistidos nos diferentes pontos da Rede de Atenção Psicossocial (RAPS). A psicoeducação é uma intervenção que promove habilidades cognitivas a partir de novas percepções de si e da realidade, fomentando a ampliação do autoconhecimento, de aspectos sobre os problemas de saúde, fenômenos mentais, sintomas e tratamentos específicos. Esta modalidade de abordagem possui relevância científica, evidenciando-se que a sua aplicação está diretamente relacionada à neuroplasticidade, com respectiva modificação do comportamento e redução de episódios recorrentes e sintomas agudos dos transtornos mentais e, subsequentemente, de internações hospitalares. Considera-se, portanto, que a psicoeducação constitui uma técnica segura de abordagem terapêutica utilizada pelo enfermeiro de saúde mental durante o seu processo de trabalho. Para tanto, sugere-se que mais investigações sobre a psicoeducação sejam 
desenvolvidas, para que se refine este arsenal científico e se possa construir novos conhecimentos no campo da enfermagem de saúde mental.

Palavras-chave: psicoeducação, enfermagem, saúde mental, psiquiatria.

\begin{abstract}
This is a narrative review of the literature, whose objective is to describe psychoeducation as a technique used in the work process of mental health nurses. Non-systematic searches for studies were carried out in April and May 2020, without time frame, in the LILACS, MEDLINE and SCOPUS databases. It was evidenced that psychoeducation is a technique used by mental health nurses in an individual format, through nursing consultation or collective, through the formation of groups. In this sense, it constitutes a relevant cognitive factor for the modification of thinking and behavior in users assisted at different points of the Psychosocial Care Network (RAPS). Psychoeducation is an intervention that promotes cognitive skills based on new perceptions of oneself and of reality, promoting the expansion of self-knowledge, aspects of health problems, mental phenomena, symptoms and specific treatments. This modality of approach has scientific relevance, showing that its application is directly related to neuroplasticity, with the respective behavior modification and reduction of recurrent episodes and acute symptoms of mental disorders and, subsequently, hospital admissions. Therefore, psychoeducation is considered to be a safe technique of therapeutic approach used by mental health nurses during their work process. Therefore, it is suggested that more investigations on psychoeducation be developed, so that this scientific arsenal can be refined and new knowledge can be built in the field of mental health nursing.
\end{abstract}

Keywords: psychoeducation, nursing, mental health, psychiatry.

\title{
1 INTRODUÇÃO
}

A enfermagem é uma profissão da área da saúde e uma ciência que estuda o cuidado humano sob diferentes perspectivas, materializando-se como trabalho que fomenta, historicamente, a preservação da vida em sua totalidade ${ }^{(1)}$. No campo da saúde mental, a enfermagem está inserida em uma equipe multiprofissional de saúde, atuando nos diferentes pontos da Rede de Atenção Psicossocial (RAPS), seja na assistência direta aos usuários ou na gestão ${ }^{(2-3)}$.

Nos segmentos da RAPS, o papel gerencial do enfermeiro de psiquiatria e saúde mental inclui o planejamento, a coordenação, a organização, a direção e a avaliação do serviço de enfermagem, com o objetivo central de garantir uma prática assistencial segura e humanizada, desenvolvida a partir de diferentes tecnologias e recursos terapêuticos ${ }^{(2)}$.

No tocante ao papel assistencial do enfermeiro em psiquiatria e saúde mental, é relevante apontar para a construção histórica do seu processo de trabalho que, paulatinamente, vem sendo considerado para ressignificar o modus operandi centrado no hospital. Atenta-se, atualmente, para uma assistência integral conforme as necessidades biopsicossociais do usuário, seja de base comunitária ou hospitalar, porém, apoiada em critérios técnicos, éticos, legais e por políticas públicas vigentes ${ }^{(4-5)}$. 
Ressalta-se, portanto, que a prática assistencial (contemporânea) do enfermeiro no campo da psiquiatria e saúde mental pode ser desenvolvida, em especial, sobre dois pilares: atuando de modo independente dos demais profissionais da equipe, através da consulta de enfermagem ${ }^{(6)}$, e/ou; sob uma perspectiva multiprofissional, por meio do Projeto Terapêutico Singular (PTS) ${ }^{(7)}$.

A consulta de enfermagem é uma atividade privativa do enfermeiro e se estrutura conforme as etapas do processo de enfermagem (histórico, diagnóstico, planejamento, implementação e avaliação de enfermagem), objetivando a prescrição de intervenções específicas à pessoa ou coletividade humana e a organização da documentação emergente da prática profissional do enfermeiro. No decorrer da consulta de enfermagem, o enfermeiro de saúde mental considera, valoriza e empodera o usuário sobre sua autonomia no movimento de promoção de saúde e construção da cidadania ${ }^{(6)}$.

$\mathrm{Na}$ consulta de enfermagem são analisadas as condições biológicas, psicológicas e sociais do usuário, por meio do exame físico, exame do estado mental (funções psíquicas), história de vida e demais informações relevantes. Além disso, avalia-se a apresentação geral e comportamento do usuário, capacidade de insight, adesão às terapêuticas prescritas, sinais e sintomas dos transtornos mentais e de intoxicação medicamentosa, vulnerabilidade social, riscos de suicídio, autoagressão, heteroagressão, quedas, entre outros ${ }^{(4)}$. Destaca-se que o exame do estado mental é constantemente realizado pelos enfermeiros de saúde mental em seu processo de trabalho, buscando analisar a consciência, atenção, orientação, memória, inteligência, linguagem, afeto/humor, vontade, psicomotricidade, sensopercepção e pensamento - juízo da realidade do usuário ${ }^{(8)}$.

Sob uma dimensão multiprofissional, o papel assistencial do enfermeiro de saúde mental se materializa na Projeto Terapêutico Singular (PTS), um processo contínuo e sistemático, deliberado às necessidades biopsicossociais e autonomia do usuário, que tem participação ativa neste processo ${ }^{(7)}$. O PTS objetiva integrar várias abordagens para efetivar a complexidade do trabalho multiprofissional de saúde e, neste sentido, cada técnico da equipe será um profissional de referência de um número específico de usuários, considerando-se o vínculo estabelecido entre eles ${ }^{(9)}$.

O PTS é construído em quatro momentos: diagnóstico (avaliações físicas, mentais e sociais do usuário, para identificar vulnerabilidades e perceber como é o seu insight, necessidades, limitações, família, trabalho e outras relações sociais); definição de metas (elaboradas a curto, médio e longo prazo, acordadas entre o usuário e o profissional de referência); divisão de responsabilidades (definidas as tarefas de cada membro da equipe, usuário e familiares), e; reavaliação (discutidas a adesão do usuário ao planejamento inicial, sua evolução e necessidade de mudanças no plano $)^{(7)}$.

Na completude da sua prática assistencial, o enfermeiro de saúde mental utiliza um arsenal técnicocientífico, além de tecnologias materiais e não materiais, para desenvolver estratégias terapêuticas e um 
cuidado profissional seguro e qualificado, seja em formato individual ou coletivo. No decorrer destes movimentos assistenciais na consulta de enfermagem ou PTS, a psicoeducação eleva-se como elemento substancial da prática profissional, pois resgata, reconhece e valoriza a autonomia do usuário sobre os combinados terapêuticos e sua vida de um modo geral.

A psicoeducação é uma técnica utilizada pelo enfermeiro no contexto da saúde mental, em perspectiva individual ou a partir da formação de grupos e, assim como a reestruturação cognitiva e o insight, caracteriza-se como fator cognitivo estratégico para a modificação do pensamento e comportamento humano ${ }^{(10)}$.

Trata-se de uma intervenção que busca promover a ampliação das habilidades cognitivas da pessoa com transtorno mental e/ou seus familiares, através da aquisição de novas percepções, ajustes de interpretações inadequadas sobre si e da realidade, suscitando um gradativo aumento do autoconhecimento, conhecimentos sobre sua condição de saúde, fenômenos mentais, sintomas e tratamentos específicos ${ }^{(10)}$.

De acordo com o desenrolar dos apontamentos supramencionados, esta revisão narrativa de literatura tem o objetivo de descrever a psicoeducação enquanto técnica utilizada no processo de trabalho do enfermeiro de saúde mental. Para tanto, justifica-se a realização do estudo, pois considera-se que ele poderá contribuir com uma lacuna de conhecimento existente neste campo de atuação da enfermagem em saúde mental. Além disso, vale ressaltar o presente estudo possui relevância para o cenário científico, por discorrer sobre questões inerentes às dimensões da psicoeducação enquanto abordagem realizada pelo enfermeiro de saúde mental.

\section{PSICOEDUCAÇÃO ENQUANTO RECURSO TERAPÊUTICO EM SAÚDE MENTAL}

Os recursos terapêuticos empregados em psiquiatria e saúde mental vêm passando por transformações histórico-sociais, em especial, a partir do investimento em pesquisas e o subsequente desenvolvimento de políticas públicas mais bem estruturadas. Outrossim, os recursos vigentes, de perspectiva multiprofissional, que incluem a farmacoterapia, tratamentos com diferentes abordagens psicoterapêuticas e outras tecnologias materiais e não materiais, ensejam maior segurança aos tratamentos oferecidos às pessoas com transtornos mentais nos diferentes pontos da RAPS.

Esta evolução é uma consequência natural da reestruturação da sociedade e acontece porque as suas necessidades não são estáticas, exigindo, de tempos em tempos, novas verdades que ressignificam as percepções de mundo pelo indivíduo. Neste movimento, é expressivo salientar que pesquisadores em saúde mental acompanham esta transformação social e tencionam novos olhares às necessidades dos usuários, incluindo observações mais abrangente sobre as diferentes formas de sofrimento humano e novos tratamentos baseados em evidências. 
A psicoeducação é emergente do campo da psicoterapia, que por sua vez, tem sua origem nas palavras gregas Psykê (mente) e Therapeuein (curar), que desde o final do século XIX, vem sendo empregada como tratamento de diferentes transtornos mentais ${ }^{(11)}$. Compreende uma técnica psicoterapêutica emergente de diferentes modelos e concepções teóricas, que buscam compreender e aplicar tratamentos não farmacológicos efetivos frente ao contexto do sofrimento mental ${ }^{(12)}$.

Sobre o termo psicoeducação, pesquisadores sugerem que ele seja dividido em duas estruturas: a primeira, "psico", faz menção a uma técnica psicoterápica e, a segunda, "educação", está relacionada à área pedagógica que destaca o processo ensino-aprendizagem. Deste modo, a utilização da psicoeducação enquanto técnica aplicada em diferentes campos da saúde, estimula o desenvolvimento social, emocional e comportamental do usuário, sendo o profissional de saúde, o agente facilitador desta transformação ${ }^{(11,13)}$.

Dentre os diferentes espaços de saúde em que a psicoeducação é desenvolvida, vale mencionar o campo de cuidados paliativos, de doenças crônico-degenerativas, de processos cirúrgicos, sofrimentos emocionais, transtornos mentais, entre outros ${ }^{(14-17)}$. Entretanto, para que uma abordagem psicoeducativa seja efetiva, é necessário que ela seja empregada por profissionais capacitados, de maneira sistemática e com o devido suporte técnico-científico ${ }^{(12-13)}$.

Do mesmo modo, para que a psicoeducação modifique o comportamento e faça uma reestruturação cognitiva eficiente, se faz necessária a habilidade de insight. Em termos conceituais, a reestruturação cognitiva é um processo de "correção de distorções cognitivas, de percepções e interpretações erradas ou distorcidas como forma de modificar o comportamento e as emoções”. O insight, por sua vez, expressa o grau de entendimento que a pessoa tem sobre o seu problema, ou seja, é quando a pessoa percebe a conexão entre emoções e comportamentos presentes e significativos na sua vida ${ }^{(10: 123)}$.

No contexto da saúde, a psicoeducação tem dois objetivos fundamentais, sendo o primeiro, aumentar o conhecimento da pessoa sobre o seu problema de saúde, sinais e sintomas dos transtornos, mecanismos envolvidos na origem e perpetuação do problema, tratamentos, prevenção de recaídas e ativação de recursos para lidar com os efeitos adversos. O segundo objetivo tenciona aproximar a pessoa da psicoterapia e estratégias disponíveis para afastar os sintomas ${ }^{(10)}$. Considera-se aqui, incluir um terceiro objetivo, onde entende-se que a psicoeducação fomenta a autonomia da pessoa sobre os aspectos decisórios comuns da sua vida.

A abordagem psicoeducativa é uma estratégia de forte evidência que evita a reagudização do transtorno mental e favorece o tratamento eficaz em condições como o transtorno bipolar, transtorno de déficit de atenção/hiperatividade, transtornos de ansiedade, e ainda previne recaídas nos quadros de dependência química ${ }^{(10)}$. É uma técnica multifacetada, considerando que ela envolve diferentes teorias e bases técnico-científicas do campo da psicologia, pedagogia e saúde, voltadas ao contexto social, 
comportamental e cognitivo das pessoas, possibilitando uma compreensão sob diferentes prismas que envolvem o adoecimento ${ }^{(18,14)}$.

Durante a sua aplicação, o enfermeiro/terapeuta deve promover a educação individual ou coletiva dos usuários e seus familiares, podendo também, fornecer material explicativo, orientar leituras, consultas à internet e outros. A psicoeducação é uma técnica bastante empregada nas sessões de terapia cognitiva, terapia comportamental, terapia interpessoal, grupos terapêuticos de autoajuda e terapias de apoio ${ }^{(10)}$.

Neste entendimento, para que haja uma maior compreensão sobre os cenários onde a psicoeducação é desenvolvida pelo enfermeiro de saúde mental, considera-se fazer menção à RAPS e aos espaços onde a assistência é realizada.

A RAPS é uma rede temática de atenção à saúde, destinada a garantir um atendimento integral às pessoas com transtornos mentais e àquelas com necessidades decorrentes do uso de drogas, através uma equipe multiprofissional de saúde. Foi estabelecida através da Portaria $\mathrm{N}^{\circ} 3.088$ de 23 de dezembro de 2011, com objetivo de construir, ampliar e articular todos os seus segmentos à atenção de pessoas com transtornos mentais e àquelas com necessidades decorrentes do uso de substâncias psicoativas (BRASIL, 2011). Sequencialmente, a Portaria GM/MS N 3588, de 21 de dezembro de 2017 e a Resolução CIT N ${ }^{\circ}$ 32/2017, de 17 de dezembro de 2017, atualizaram seus pontos de atenção ${ }^{(3)}$.

As diretrizes que constituem as bases fundamentais da RAPS incluem o respeito aos direitos humanos, assegurando a autonomia e liberdade das pessoas, refutando os preconceitos e estigmas sociais; isonomia de uma assistência multidisciplinar para a prática de um cuidado integral com consequente redução de danos; desenvolvimento de ações humanizadas e centradas na pessoa, impulsionando a prática da inclusão social e o exercício da cidadania; políticas de educação permanente em saúde; e, elaboração de estratégias de cuidado em serviços de base territorial e comunitária, que permita a participação dos usuários e seus familiares na construção do $\operatorname{PTS}^{(19)}$.

Atualmente, esta RAPS encontra-se organizada na Atenção Básica em Saúde (Unidade Básica de Saúde, Consultório de Rua, Centro de Convivência); Atenção Psicossocial Especializada (Centro de Atenção Psicossocial - CAPS em diferentes modalidades - CAPS I, II e III, CAPSi, CAPSad, CAPSad III e IV); Atenção de Urgência e Emergência (Serviço de Atendimento Móvel de Urgência - SAMU, sala de estabilização, UPA 24 horas, portas hospitalares de atenção à urgência/pronto socorro); Atenção Residencial de Caráter 16 Transitório (Unidades de Acolhimento Adulto e Infanto-juvenil, Serviços de Atenção em Regime Residencial); Atenção Hospitalar (Hospital Dia, Unidades de Referência Especializadas em Hospitais Gerais, Hospital Psiquiátrico Especializado, Ambulatório de Saúde Mental); Estratégias de desinstitucionalização (Serviços Residenciais Terapêuticos (SRT) I e II); Reabilitação Psicossocial $^{(19,3)}$. 
Em cada ponto de atenção desta rede temática, os enfermeiros assistirão pessoas com transtornos mentais diversos, sob diferentes condições socioeconômicas e que apresentam necessidades dessemelhantes. Portanto, ações psicoeducativas podem ser utilizadas como estratégias de grande valia para promoção de saúde.

\section{PSICOEDUCAÇÃO E NEUROPLASTICIDADE}

A psicoeducação objetiva tratar problemas de natureza emocional, cognitiva e comportamental, modificando-os. Ela se caracteriza como recurso que identifica o homem enquanto um ser passível de transformação biológica e psicológica, a partir da exposição a distintas experiências ${ }^{(10)}$.

Nesta dimensão e para uma compreensão processual da relação mente, cérebro e aprendizado, fazse referência ao dualismo, que defende a mente e o cérebro como estruturas separadas, e ao monismo, que considera as duas estruturas unificadas, sendo a mente um produto da atividade do cérebro ${ }^{(10)}$.

Kandel (1998) sintetizou a relação entre mente, cérebro e aprendizado em cinco princípios: 1) Todos os processos mentais derivam de operações do cérebro; 2) O material genético exerce grande influência sobre o comportamento; 3) Fatores sociais podem modificar o comportamento por meio de alterações na expressão gênica; 4) Alterações na expressão genética induzidas pelo aprendizado promovem mudanças nas conexões neurais, e; 5) A psicoterapia altera a expressão gênica, a forma e a função cerebral ${ }^{(20,10)}$.

Com base nas considerações ora descritas, é sugestivo estabelecer uma relação entre psicoeducação e neuroplasticidade, que por sua vez, é um conceito orientado para a explicação da relação entre fatores sociais, aprendizado e cérebro ${ }^{(21,22)}$. A neuroplasticidade, portanto, é percebida como a habilidade que o cérebro tem de transformar-se e adaptar-se, a partir de sucessivas interações e estímulos impostos pelo ambiente e experiência ${ }^{(21)}$.

Mediante estudos no campo das neurociências, verificou-se que o tecido nervoso tem a capacidade para transformar-se ao longo da vida, frente a exposição às experiências, caracterizando-se como ponto de partida e eixo central estimulante ou limitante da plasticidade neuronal ${ }^{(23)}$.

Associado à experiência, o aprendizado e a memória estão relacionados às modificações sinápticas e ao fortalecimento destas vias, mostrando-se articulados à construção de novas sinapses e ao recrutamento de neurônios próximos, para formar novas conexões ${ }^{(8)}$. Esta habilidade cerebral abarca um campo de conhecimento que está vinculado ao comportamento e aprendizado, enfraquecimento ou fortalecimento de sinapses e até a neurogênese ${ }^{(24)}$. Desta forma, é premente sinalizar que a aplicação da psicoeducação está relacionada ao desenvolvimento da neuroplasticidade e respectiva modificação do comportamento ${ }^{(8)}$.

Estudos apontam que os benefícios da psicoeducação incluem a melhor adesão do usuário à terapêutica prescrita, redução do número de internações hospitalares e uma menor ocorrência de alterações 
episódicas de humor ${ }^{(25)}$. Uma investigação realizada na cidade de Barcelona em 2003, identificou que a psicoeducação associada à farmacoterapia reduziu significativamente o número de novos episódios de mania em usuários, durante um acompanhamento de dois anos com equipe multiprofissional. Em uma amostra de pessoas com depressão, durante o mesmo período supramencionado, observou-se menor ocorrência dos sintomas após intervenções psicoeducativas ${ }^{(26)}$.

Outra publicação evidenciou que os benefícios da psicoeducação tendem a durar longos períodos, diminuindo assim, os episódios recorrentes de reagudização do transtorno mental, fato este que impacta diretamente na redução de custos na área da saúde ${ }^{(27)}$. Deste modo, percebe-se que a psicoeducação é uma intervenção de forte impacto na área da saúde mental, atuando como coadjuvante a outras formas de tratamento dos transtornos mentais.

\section{A ABORDAGEM PSICOEDUCACIONAL NO PROCESSO DE TRABALHO DO ENFERMEIRO DE SAÚDE MENTAL}

O processo de trabalho nos núcleos operacionais da RAPS é multiprofissional e o enfermeiro integra a equipe nos diferentes segmentos da rede, assumindo papel de protagonismo, seja na gestão dos serviços ou na assistência direta aos usuários e seus familiares. Nesta direção, o Conselho Federal de Enfermagem (COFEN), através da Resolução N 678/2021, aprova a atuação da equipe de enfermagem para atuar no contexto de trabalho em saúde mental e psiquiatria ${ }^{(2)}$.

Sob a perspectiva da gestão do cuidado realizado pelo enfermeiro na RAPS, existe uma reflexão que explora a compreensão dicotômica entre os processos de trabalho assistenciais e gerenciais, possivelmente relacionados à divisão técnica e histórica do trabalho da profissão, que dissociou o trabalho intelectual do trabalho manual ${ }^{(28)}$. Os enfermeiros de saúde mental atuam nos diferentes pontos da RAPS em funções assistenciais e gerenciais que permitem articular os serviços, além de ressignificar as dimensões estratégicas, relacionais e conceituais da sua prática, o que resulta em um cuidado profissional mais seguro e apoiado em preceitos éticos, técnicos e legais.

Dentro das esferas assistenciais do campo da psiquiatria e saúde mental, o enfermeiro utiliza a psicoeducação enquanto abordagem terapêutica às pessoas com transtornos mentais. Entretanto, para que o profissional desenvolva ações psicoeducativas com segurança em sua prática, ele constrói um arsenal científico a partir de diferentes áreas de atuação. Esta construção é essencial para que se realize uma abordagem segura nas diferentes fases do transtorno mental, buscando promover a saúde e melhorar a adesão terapêutica e prognóstico do usuário.

Em sua prática, o enfermeiro de saúde mental desenvolve o seu processo de trabalho a partir da Sistematização da Assistência de Enfermagem, que segundo a Resolução No 358/2009 do COFEN, é uma 
metodologia que organiza o trabalho profissional quanto ao método, pessoal e instrumentos, tornando possível a operacionalização das etapas do Processo de Enfermagem ${ }^{(6,2)}$.

A modalidade de consulta de enfermagem é uma atividade privativa do enfermeiro (Resolução No 606/2019 do COFEN) para identificar os problemas de saúde, prescrever e implementar intervenções de enfermagem que visam promover, proteger, recuperar e reabilitar o usuário ${ }^{(29)}$. Ela inicia-se pelo histórico de enfermagem, que é uma construção sistemática e contínua, o enfermeiro levanta informações biopsicossociais sobre o usuário, para a definição dos diagnósticos de enfermagem. Em seguida realiza-se o planejamento assistencial, define-se os resultados esperados e implementa-se as intervenções de enfermagem. Após estes procedimentos faz-se a avaliação de enfermagem ${ }^{(6)}$.

Durante estas etapas inter-relacionadas o enfermeiro prescreve e executa abordagens psicoeducativas que podem ser realizadas em formato individual (durante a consulta de enfermagem) ou grupal (na formação de grupos).

Igualmente relevante, durante a implementação do PTS, que é um conjunto de propostas e condutas terapêuticas articuladas para um indivíduo, uma família ou um grupo que resulta da discussão coletiva de uma equipe interdisciplinar ${ }^{(19)}$, o enfermeiro também planeja e desenvolve a psicoeducação em caráter individual ou em formato de grupo.

A formação de grupos psicoeducativos é uma ferramenta comumente aplicada no processo de trabalho do enfermeiro de psiquiatria e saúde mental. Para realização dos grupos, são usadas técnicas específicas de diferentes modelos psicoterapêuticos e fatores terapêuticos comuns. Os fatores terapêuticos abarcam elementos da psicoterapia, que contribuem para melhorar a condição do usuário e familiar, "proveniente tanto das ações do terapeuta quanto das ações dos integrantes do grupo ou do próprio usuário"(10:441).

Os fatores terapêuticos de um grupo, em geral, incluem:

1. Instilação de esperança: trata-se da expectativa de conseguir melhorar, onde a esperança é considerada um impulso para a modificação do comportamento;

2. Universalidade: a promoção do alívio do usuário acontece por não sentir-se sozinho; 3) Orientação: acontece o reconhecimento do significado do problema e funcionamento psíquico através da psicoeducação;

3. Altruísmo: identificar o sentimento do outro e proporcionar apoio mútuo;

4. Reedição familiar: o usuário é estimulado a reviver conflitos de família;

5. Aprendizagem interpessoal - output: corresponde ao desenvolvimento de novas habilidades sociais através da convivência em grupo; 
6. Aprendizagem interpessoal - input: viabilização do relacionamento interpessoal entre os membros do grupo e correção da experiência emocional por meio da exposição do usuário a situações que ele não conseguiu enfrentar anteriormente;

7. Identificação: modelar-se com base nos aspectos dos outros membros do grupo e do terapeuta;

8. Coesão: significa o bom relacionamento e aceitação entre os membros, incluindo o terapeuta.

9. Catarse: possibilidade de alívio pela exposição de sentimentos;

10. Fatores existenciais: aspectos relacionados à existência e à confrontação da condição humana, como mortalidade, responsabilidade e liberdade;

11. Autocompreensão: proposta viabilizar a aceitação do usuário de aspectos sobre si antes negados, possibilitando um resgate de questões reprimidas e rejeitadas por eles mesmos ${ }^{(30)}$.

Os grupos terapêuticos ou operativos têm como objetivo fundamental, fortalecer a saúde mental dos usuários, em aspectos físicos e psicossociais. A formação de um grupo pode variar conforme os objetivos e finalidades, embora existam características gerais em comum. Neste sentido, para que os benefícios desta técnica ocorram, deve ser considerado o planejamento em relação à estrutura do grupo e das sessões ${ }^{(10,31)}$.

Outrossim, para que os benefícios da terapia de grupo ocorram, é relevante considerar os seguintes pontos da sua elaboração: a) Planejamento: finalidade do grupo; para quem o grupo se destina; tipo de grupo (como ele funcionará) - homogêneo ou heterogêneo; aberto ou fechado; enquadramento ou setting soma de todos os procedimentos que organizam, normatizam e possibilitam o funcionamento do grupo; número de participantes, número de reuniões, tempo de duração ${ }^{(10,31)}$; b) Seleção: motivação (reconhecimento da necessidade de tratamento e da disposição para mudanças); composição homogênea (participantes com alguma característica em comum); composição heterogênea ${ }^{(10,31)}$.

Neste entendimento, o coordenador do grupo de psicoeducação precisa compreender a fundamentação teórica e as técnicas específicas para realização desta estratégia. Entretanto, destaca-se que, além dos conhecimentos científicos, a cordialidade e a empatia exercem papéis expressivos e influenciam a motivação e adesão dos participantes. Conforme sua atuação no grupo, o terapeuta pode suscitar a participação e colaboração ativa (ou não), gerando respostas adaptativas. O terapeuta também estimula a construção de um ambiente harmônico e tolerante frente as diferenças individuais, encorajando os participantes a falar abertamente ${ }^{(30)}$.

\section{CONSIDERAÇÕES FINAIS}

Ampliar a compreensão acerca das nuances da psicoeducação enquanto abordagem terapêutica utilizada por enfermeiros de saúde mental demonstrou a relevância, amplitude e pluralidade da temática. 
Com inclinação correspondente, o movimento orientado à percepção atenta desta técnica, abriu espaços para reflexionar sobre os limites e possibilidades das estratégias psicoeducativas utilizadas por enfermeiro deste campo de atuação.

A psicoeducação é uma abordagem terapêutica empregada por enfermeiros de saúde mental em todo o seu processo de trabalho, nos diferentes pontos da RAPS, seja no formato individual, através da consulta de enfermagem e/ou PTS e a partir de formação de grupos. Nesta linha de atuação, tanto a psicoeducação como a reestruturação cognitiva e o insight, constituem um fator cognitivo estratégico para a modificação do pensamento e comportamento das pessoas com transtornos mentais.

A partir da consulta de enfermagem e do PTS, o enfermeiro coleta dados relevantes para o planejamento da assistência e faz o levantamento dos diagnósticos para, posteriormente, implementar ações de enfermagem que serão realizadas durante a sua prática assistencial. Dentre elas, estão as estratégias psicoeducativas que consideram orientar o usuário para que o seu comportamento esteja alinhado às práticas de promoção da sua saúde.

A psicoeducação também é relevante, por sua relação direta com a neuroplasticidade, que modifica o comportamento e reduz os episódios agudos e recorrentes dos transtornos mentais e a subsequente internação hospitalar.

De acordo com as discussões elencadas ao longo do estudo, foi evidenciado que a psicoeducação promove a autonomia do usuário sobre o seu processo de saúde e transtorno mental, pois favorece o aumento das habilidades cognitivas a partir de novas percepções construídas. Outrossim, as estratégias psicoeducativas ainda proporcionam ampliação do autoconhecimento, conhecimento sobre o transtorno mental, sinais e sintomas agudos, tratamentos específicos, fenômenos mentais e prevenção de recaídas.

Neste movimento, conclui-se que a psicoeducação é uma técnica segura utilizada pelo enfermeiro de saúde mental em sua prática assistencial. Entretanto, sugere-se que novas investigações sobre a psicoeducação no contexto da enfermagem em saúde mental sejam realizadas, suscitando a produção novos conhecimentos neste campo de atuação profissional. Outrossim, sinaliza-se que a realização deste estudo pode contribuir para uma lacuna de conhecimento existente nesta área, além de fomentar o interesse de outros enfermeiros sobre a realização de investigações sobre psicoeducação. 


\section{REFERÊNCIAS}

1. Pires, D. A enfermagem enquanto disciplina, profissão e trabalho. Revista Brasileira de Enfermagem, [S.L.], v. 62, n. 5, p. 739-744, out. 2009. FapUNIFESP (SciELO). http://dx.doi.org/10.1590/s003471672009000500015 .

2. COFEN. Conselho Federal de Enfermagem. Resolução n. 678, de agosto de 2021. Aprova a atuação da Equipe de Enfermagem em Saúde Mental e em Enfermagem Psiquiátrica. Diário Oficial da União, Brasília: COFEN, 2021. Disponível em http://www.cofen.gov.br/resolucaocofen-no-678-2021_90358.html

3. BRASIL. Ministério da Saúde. Portaria n. 3.588, de 21 de dezembro de 2017. Altera as Portarias de Consolidação no 3 e n ${ }^{\circ}$ 6, de 28 de setembro de 2017, para dispor sobre a Rede de Atenção Psicossocial, e dá outras providências. Disponível em: http://bvsms.saude.gov.br/bvs/saudelegis/gm/2017/prt3588_22_12_2017.html. Acesso em 8 de março de 2021.

4. Bruggmann, M. S.; Souza, A. I. J.; Costa, E. Schneider, D. G.; Schmitz, E. L.; Mazera, M. S. Construção de um saber coletivo para implantação do processo de Enfermagem em um hospital psiquiátrico especializado. REME - Rev Min Enferm. 2019;23:e-1270. DOI: 10.5935/1415-2762.20190118

5. Amarante, P. Nunes M. O. A reforma psiquiátrica no SUS e a luta por uma sociedade sem manicômios. Ciência \& Saúde Coletiva, 23(6):2067-2074, 2018. [citado em 2019 abr. 21];23(6):2067-74. Disponível em: http://dx.doi.org/10.1590/1413-81232018236.07082018

6. COFEN. Conselho Federal de Enfermagem. Resolução Nº. 358/2009. In: Portal do COFEN. Disponível em: http://www.cofen.gov.br/resoluo-cofen-3582009_4384.html. Acesso em: 10 de março de. 2021.

7. BRASIL. Ministério da Saúde. Secretaria de Atenção à Saúde. Núcleo Técnico da Política Nacional de Humanização. Clínica ampliada, equipe de referência e projeto terapêutico singular / Ministério da Saúde, Secretaria de Atenção à Saúde, Núcleo Técnico da Política Nacional de Humanização - 2. ed. - Brasília, 2007.

8. Dalgalarrondo, P. Psicopatologia e semiologia dos transtornos mentais. 3. ed. Porto Alegre: Artmed, 2019. $440 \mathrm{p}$.

9. Campos, G. W. S.; Amaral, M. A. A clínica ampliada e compartilhada, a gestão democrática e redes de atenção como referenciais teórico-operacionais para a reforma do hospital. Ciência \& Saúde Coletiva, v. 12, n. 4, p. 849-859, ago. 2007. FapUNIFESP (SciELO). http://dx.doi.org/10.1590/s141381232007000400007.

10. Cordioli, A.V.; Grevet, E. H. Psicoterapias: abordagens atuais. 4. ed. - Porto Alegre: Artmed, 2019.

11. Osório F. L.; Silva, U. C. A., Mendes, A.I. F.; Pavan-cândido, C. C. Psicoterapias: conceitos introdutórios para estudantes da área da saúde. Medicina (Ribeirão Preto, Online.) 2017;50(Supl.1),janfev.:3-21. http://dx.doi.org/10.11606/issn.2176-7262.v50isupl1.p3-21.

12. Wood, M. M.; Brendro, L. K.; Fecser, F. A.; Nichols, P. (1999). Psychoeducation: An Idea Whose Time Has Come. Richmond, VA: The Council for Children with Behavioral Disorders. 
13. Menezes, S. L. Souza, M. C. B. M. Grupo de psicoeducação no transtorno afetivo bipolar: reflexão sobre o modo asilar e o modo psicossocial. Revista Escola de Enfermagem USP, 45(4), 996-1001. doi:10.1590/S008062342011000400029

14. Lemes C. B; Neto, J. O. Aplicações da Psicoeducação no Contexto da Saúde. Trends in Psychology / Temas em Psicologia - Março 2017, Vol. 25, nº 1, 17-28 DOI: 10.9788/TP2017.1-02.

15. Alcântara, T. V.; Shioga, J. E. M.; Lima, M. J. V.; Lage, A. M. V.; Maia, A. H. N. (2013). Intervenções psicológicas na sala de espera: Estratégias no contexto da oncologia pediátrica. Sociedade Brasileira de Psicologia Hospitalar, 16(2), 103-119.

16. Andretta, I.; Oliveira, M. S. (2011). A entrevista motivacional em adolescentes usuários de droga que cometeram ato infracional. Psicologia: Refl exão e Crítica, 24(2), 218-226.

17. Gonçalves-Pereira, M.; Sampaio, D. (2011). Psicoeducação familiar na demência: da clínica à saúde pública. Revista Portuguesa de Saúde Pública, 29(1), 3-10. doi:10.1016/S08709025(11)70002-5

18. Rahmani, F.; Ranjbar, F.; Ebrahimi, H.; Hosseinzadeh, M. (2015). The effects of Group Psychoeducational Programme on attitude toward mental illness in families of patients with schizophrenia, 2014. Journal of Caring Sciences, 4(3), 243-251.

19. BRASIL. Ministério da Saúde. Portaria GM/MS n. 3.088, de 23 de dezembro de 2011. Institui a Rede de Atenção Psicossocial para pessoas com sofrimento ou transtorno mental e com necessidades decorrentes do uso de crack, álcool e outras drogas, no âmbito do Sistema Único de Saúde (SUS). Diário Oficial [da] União, Brasília, DF, 24 dez. 2011. Disponível em: bvsms.saude.gov.br/bvs/saudelegis/gm/2011/prt3088_23_12_2011_rep.html. Acesso em: 10 março de 2021.

20. Kandel, E.R. A new intellectual framework for psychiatry. Am J Psychiatry. 1998;155(4):457-69.

21. Kendler, K. S. What psychiatric genetics has taught us about the nature of psychiatric illness and what is left to learn. Mol Psychiatry. 2013;18(10):1058-66.

22. Bruggmann, M. S. Psicoterapia, neuroplasticidade e o funcionamento cerebral. Archives of Health, Curitiba, v.2, n.1, p.106-117, jan./feb.,2021. ISSN 2675-4711. DOI: 10.46919/archv2n1-009

23. Arteni, N. S.; Alexandre Netto. C. Neuroplasticidade. In: Kapczinski F.; Quevedo, J.; Izquierdo I. Bases biológicas dos transtornos psiquiátricos. Porto Alegre: Artmed, 2004.

24. Gulyaeva N. V. Molecular mechanisms of neuroplasticity: an expanding universe. Biochemistry. 2017;82(3):237-42.

25. Scott, J. (2003). Group psychoeducation reduces recurrence and hospital admission in people with bipolar disorder. Evid Based Ment Health;6(4):115.

26. Colom, F.; Vieta, E. Improving the outcome of bipolar disorder through nonpharmacological strategies: the role of psychoeducation. Rev Bras Psiquiatr 26(3):47-50, 2004. 
27. Colom, F.; Vieta, E.; Sanchez-Moreno, J.; Martinez-Aran, A.; Torrent, C.; Reinares, M. et al. Psychoeducation in bipolar patients with comorbid personality disorders. Bipolar Disord 6(4):294-298, 2004.

28. Santos, J. L. G.; Erdmann, A. L.; Meirelles, B. H. S.; Lanzoni, G. M. M.; Cunha, V. P.; Ross, R. Integração entre dados quantitativos e qualitativos em uma pesquisa de métodos mistos. Texto \& Contexto - Enfermagem, [S.L.], v. 26, n. 3, p. 1-9, 2017. FapUNIFESP (SciELO). http://dx.doi.org/10.1590/010407072017001590016.

29. COFEN. Conselho Federal de Enfermagem. Resolução No ${ }^{\circ}$ 606/2019. In: Portal do COFEN. Disponível em: http://www.cofen.gov.br/resolucao-cofen-no-606-2019_70088.html. Acesso em: 12 de março de. 2021.

30. Yalom I. D.; Leszcz, M. Psicoterapia de grupo: teoria e prática. 5. ed. Porto Alegre: Artmed; 2006.

31. Zimerman, D. E. A importância dos grupos na saúde, cultura e diversidade. Vínculo. Vínculo. 2007;4(4):1-16. 